\title{
Results Based Management (RBM): An antidote to pro- gram management
}

\author{
Bongs Lainjo * \\ Cybermatic International, Montreal, Canada
}

\author{
Keywords \\ Result-based management \\ Theory of change \\ United nation organization \\ Logical framework \\ Project cycle management \\ Risks and assumptions \\ Inclusive \\ Participatory \\ Environment
}

Received: 8 December 2018 Accepted: 15 January 2019 Published: 20 February 2019

\begin{abstract}
This study explores Result Based Management (RBM) in the strategic system approach by analyzing a life case study. It is a management approach purely shaped by the results. This study discusses the RBM logical framework and the theory of change. The logical framework of RBM resides on the structured, logical model, which identifies the expected outputs and, consequently, the inputs and activities required to accomplish the outcomes. The logical framework is structured around five items: assess, think, plan, do and review. The philosophical assumptions underlying this study are based on critical realism. This study seeks to establish the mechanisms applied in RBM and the required structures via a life case study of the United Nations Organizations. In critical realism ontology, three aspects are considered, the real, the actual, and the empirical. This study combines all three aspects of insightful analysis. The study notes the existence of RBM on policy papers, but there is a minimal impetus to implement it practically despite its potential benefits in achieving organizational performance. There is an inadequate degree of inclusivity, participatory, and enabling environment in occasional cases where the framework is implemented. The life case study focused on the United Nations Organization and the modalities for the implementation of RBM. In conclusion, this work has explored the concept of RBM as a management approach that targets improving results in a three-thronged model constituted of short-, medium-, and long-term types of results, outputs, outcomes, and impacts. The concept is particularly prevalent in public and not-for-profit organizations. The study has provided valuable insight into the RBM in program management and its application in a selected case study.
\end{abstract}

(C) 2019 The Author(s). Published by TAF Publishing.

\section{INTRODUCTION}

The concept of RBM is not new. Indeed, it underlies all the efforts humans consciously undertake to achieve desired results. For example, countries go to war to win; parents bring up their children to be law-abiding citizens; children go to school (elementary, middle school, high school, community college, university) to successfully graduate; farmers plant crops in anticipation of good harvests; people go to gyms to lose excessive weight; businesses are established in order to make profits; and so on. All these interventions have in common the objective of transforming inputs into intended results (outputs, outcomes, and goals) or Strategic Objectives (SO). The universality of this concept makes it a useful basis for efforts to streamline and optimize proposed interventions. From a program development perspective, definitions have limited RBM to the key results presented in a relevant Program Design Framework (PDF). This approach, while correct seems to limit the importance and significant scope of RBM. In this book, the definition of RBM has intentionally been expanded. It has also been deliberately presented from a generic perspective. Such a strategy creates room for every stakeholder and facilitates portability-lessons learned. It also enhances the versatility of this concept.

Thus, I have attempted to define a more holistic meaning of RBM. RBM can be considered as a hierarchical framework

\footnotetext{
* corresponding author: Bongs Lainjo
}

†email: bsuiru@bell.net 
of mutually complementary components (program design framework, Monitoring, and Evaluation (M\&E), Data Management (DM) and Management Information System (MIS) with synergistic dynamics that collectively yield intended or unintended objectives. The beauty of RBM is that there is no absolute "goal standard". The unavailability of an acceptable "turn-key" system is a confirmation of the dicey nature of RBM.

Each intervention is different, and as such, RBM serves as a unique framework with a common potential of promoting efficiency and accountability (effectiveness). Effective and evidence-based performance monitoring contribute substantially to the achievement of RBM. It is my hope that such a document will serve a wide audience on the one hand and facilitate program design and implementation on the other. While the target audience remains quite broad-bilateral and multilateral agencies, academic institutions, and Program Implementing Partners (PIP) - it is my hope that this document can also be used by anyone interested in an improved understanding of the concept of RBM. To this end, I have tried to minimize utilization of technical jargon as much as possible. Such an attempt will hopefully expand the target audience base and generate more interest, especially amongst the members of the program development communities. Finally, the current requirement for accountability and results by donors has provided compelling evidence of the need for information of this kind.

According to the Global Affairs Canada (2008), RBM is an approach led by a life cycle that embeds strategic thinking with people, resources, different processes, and measurements meant to improve accountability, decision-making, and transparency (Farrell, 2009; Layyinaturrobaniyah, Masyita, \& Sekartadjie, 2016; Visser, 2016). It is important to emphasize that RBM is exercised with sound judgment in an attempt to comply with all government and organizational regulations and requirements that produce maximum potential and best accountability standards. The approach is focused on results, measuring performance, adapting and learning to a new environment, and reporting on these experiences about performance (Farrell, 2009; Willy, 2017). Therefore, RBM is meant to define realism, program identification, and progress monitoring that become linked to the overall success of the program, measured through results and utilized resources, and noted by indicators appropriate to the goal (Organisation for Economic Co-operation and Development, 1997). Risks are managed under RBM, knowledge is attained, and new decisions made based on this methodology. Monitoring and evaluation, data management and management information system are in- valuable components of a robust RBM (Kusek \& Rist, 2004). The goal of RBM is to maximize the results; the effectiveness of the method is based on the outcomes, and the outcomes vary depending on the setting of the program. Nevertheless, the overall goal is to attain the results planned before the commencement of the cycle. It is about change; changing assumptions, studying changed risks and identifying changing factors (internal and external) that may interfere with the development of the program (Kusek \& Rist, 2004). Inherent in the program that utilizes RBM, is the idea that something must change to maximize potential or results. The design, then, is based on knowledge learned from experiences, that could lead to the design of a better, more effective management plan with new research, evaluative methods, practice policies, and learned lessons (Due, 2016; Kusek \& Rist, 2004). RBM entails the development of theories and ideas that reflect the process' overall goals, systemically mapping the sequence that would logically lead to the best outcome for the project (Bester, 2012; Kusek \& Rist, 2004). Otherwise defined as the Results Chain (RC), it is a visual manifestation of the relationships that must intertwine for a goal to be achieved, breaking it down into activities, inputs, outputs, and outcomes that will benefit the organization, project, program or company (Bester, 2012). Each organization will create its RC, unique and specific to its goals. For instance, the Global Affairs Canada organization has six different levels of its RC, i.e., inputs, activities, outputs, immediate outcomes (short-term), intermediate outcomes (medium-term), and outcome (long-term). In other organizations, such as the Organization for Economic Co-operation and Development (OECD), only five RC levels are defined, i.e., inputs, activities, outputs, outcomes, and impact (Organisation for Economic Co-operation and Development, 1997).

\section{Setting}

Sustainable Program Management (SPM) is a complex, process-driven framework analogous to the CARROT-BUS model, where effective management produces useful and effective outcomes, intended or unintended. SPM has, over time, been linked to sustainability as observed in Silvius (2015). Although defining the concept of sustainability is more challenging than expressing it, it has gained a foothold in program management in the recent past (Panti \& Gempes, 2018; Silvius, 2015). This book adopts the definition of sustainability as a normative concept, which is delimited by values highlighting the attitudes and behaviour of individuals, where the focus is to minimize risks in program management (Eskerod \& Huemann, 2013; Montasser \& El-Nakeeb, 
2017).

Where does RBM fit in the CARROT-BUS framework? RBM is an integrated component of every step of the ladder. It serves as a required, necessary, and sufficient revolving strategy that effectively establishes the causal links amongst the different levels of the CARROT-BUS pyramid. As already discussed in detail, in sustainable program management, the CARROT-BUS model seeks to create an enabling environment through a pyramid-structured model (Silvius, 2015). The pyramid of sustainable program management consists of six elements, namely capacity building, accountability, resources, results, ownership, and transparency. These elements of the pyramid are executed in an ascending order up the pyramid. They include the strengthening of capacity, (C); building in organization management, improving accountability, (A); effective utilization of Resources, (R); so as to achieve relevant results, (R); establishment of ownership, (O); and the achievement of transparency, (T).

Sustainable program management fits into the pyramid model, where capacity development is at the base of the pyramid. Capacity development is related to human resource development in education and training. In Groot et al. (2000), capacity development has been defined as the development of knowledge, skills, and attitude in either individuals or groups with the goal of improving management, as well as the maintenance of organization and operational infrastructures. It is the improvement of employees' ability to perform their duties and responsibilities within the organization (Groot et al., 2000).

The concept of results-based management is a management approach oriented towards results. The management approach has its roots in the public sector reforms of the 1990s, which responded to the results-driven approach as a result of economic, social, and political pressure (Pollitt \& Bouckaert, 2004). In program management, results are placed at the center of the cycle of the project from strategic planning, implementation, monitoring, and evaluations as well as reporting and the decision-making process. One of the prominent aspects of RBM is performance measurement.

As highlighted in Binnendijk (2000), performance measurement seeks to assess how a firm meets its primary aims. Performance measurement seeks to improve management through learning, and at the same time leverage it with improved decision-making and planning. To implement performance assessment, RBM relies on an external and internal pool of accountability to the firm's results (Vahamaki, Schmidt, \& Molander, 2011). Results in RBM are classified into three distinct phases-immediate outputs, intermediate outcomes, and finally, the long-term impacts. The existence of results in three different levels raises the challenge of assessment as observed by Binnendijk (2000).

In brief, results-based management is a management tool, which is based on performance management. It defines the expected results from key stakeholders in the program management process. It is a RC focusing on human and financial resources to generate both short-term and longterm outputs for the organizations. RBM is a philosophy that focuses on achieving tangible changes (Aly, 2015). It is a set of tools employed in program management that seeks to summarize organizational core objectives. Program management is focused on the achievement of defined results. In the strategic systems approach, RBM is a results-focused approach. RBM focuses on performance and achievements, which are weighed regarding impacts, outcomes or outputs (Aly, 2015).

\section{RBM BACKGROUND}

\section{Results-Based Management Logical Framework}

The logical framework of RBM resides on the structured, logical model, which identifies the expected outputs and consequently the inputs as well as activities required to accomplish the outcomes (Aly, 2015). The logical framework is structured around five items; assess, think, plan, do, and review.

Logical framework in RBM assess the current situation; what causes it or what is involved and what is going to be achieved; the plan on what to do and when and the resources involved; how it is going to be done and what are the adaptation plans; and finally reviews what was done well and what requires revision in the next period (Aly, 2015). The process of RBM is an iterative undertaking that relies on two concepts - strategy, and expected results. The process is composed of at most 12 phases, where the first seven phases are linked to result-oriented planning.

The first phase is the analysis of the problem to be addressed and its causes and effects. The second phase is the identification of principal stakeholders and beneficiaries. This stage also involves the identification of objectives and the design of interventions to meet the expected needs (Diamond, 2005). Thirdly, the expected measurable results are formulated. The performance indicators for the expected results are specified in the fourth phase. The fifth step is the setting of targets and benchmarking each indicator with the specified results to be achieved.

The sixth phase of organizational RBM is the strategy development, where a conceptual framework is drawn from 
the expected results. It is at this stage where the main modalities of actions involving the constraints and opportunities are identified (Diamond, 2005). The seventh phase is the balancing of the expected results and strategy with the available resources in the program management. The eighth phase entails management and monitoring the progress of RBM, with appropriate performance monitoring systems.

The last four phases of the application of RBM entail reporting and self-evaluation, where results are compared to the targets and the actual results achieved. Also, there is the integration of the lessons drawn and the self-evaluation, where information emanating from the monitoring system is interpreted for possible inferences and discrepancies between the expected and the achieved (Diamond, 2005). The dissemination and discussion of results and lessons are performed in a transparently and iteratively (Diamond, 2005). The performance information from the monitoring and evaluation systems is then applied in the internal program management learning and decision-making process. The significance of RBM in an organization rests in its ability to change the organization's culture by keeping track of outcomes such as financial performance and market share. One of the principles of RBM is the results chain, shown below. Resources/inputs, activities, outputs, outcomes, impact Result chain is composed of inputs, activities, outputs, outcomes, and impact. Positive or even negative effects of an intervention lead to tangible results, which are grouped into three levels as outputs, outcomes, and impacts. Outputs are the direct product and services arising from an intervention. They are the immediate effects of an intervention where one has the highest level of control (Flint, 2002). Outcomes are the medium-term effects of intervention outputs. In program management, there is lesser control of this second level of results compared to the first level, outputs. They represent the tangible changes being brought into the program management dynamics. The third level of results is the impact, which can be categorized as primary and secondary effects and can either be negative or positive (Flint, 2002). This is a sum of some precise activities and other compounding factors. This level of results has the lowest level of control. Inputs are used to perform activities; activities produce precise outputs, outputs produce outcomes, and the resultant outcomes contribute to impacts. In the RBM logic, interventions are in the form of hierarchies, which are a set of inputs and activities leading to results in the form of outputs, outcomes, and impacts (Flint, 2002).

Firstly, the implementation of RBM is dependent on the ex- tent to which the key performance measures are connected to the existing strategic or policy framework. According to Organisation for Economic Co-operation and Development (1997), successful implementation of RBM takes the time to develop its core indicators and streamline the management systems before collecting any performance data. Poate (1997) insists that organizations need to be persistent and patient because building consensus and maintaining the momentum is crucial to success. Although it may be tempting to rush the implementation, firms must come to terms that rushing implementation only serves to decrease the likelihood of having a useful measurement system (Hatch \& Cunliffe, 2006). It is worth noting that organizations are often limited in their use of indicators in several cases until they acquire sufficient measurement experience. In this regard, Itell (1998) concludes that outcomeoriented measures should be allocated sufficient time to develop them. This is bound to occur when relevant departments gain enough experience to identify both the cause and the effect. Diamond (2005) has reported that unrealistic projections of what RBM can accomplish in an organization only serve to undermine the RBM initiative. Therefore, it is the responsibility of senior managers to set and manage these expectations in their organizations.

The experiences of some major multinationals suggest some key guidelines for defining performance indicators and measures. For instance, Gibson and Boisvert (1997) argue that it is important to start with the end in mind, and this means going back to the broad objectives and vision that defined the long-term effects that the program, policy or service was intended to achieve (Downey, 1998). From this point, it becomes easy to identify both the shortterm and the medium-term results that can contribute to the achievement of the anticipated effects. It also becomes possible to identify the indicators that reflect the short-term and long-term results (Kettl, 1997). When it comes to the importance of management culture in RBM, European Commission (1999) argue that RBM requires more than just the adoption of new operational and administrative systems. Rather, there should also be an emphasis on outcomes that need a performance-oriented management culture that can support and encourage the utilization of the new management approaches. These observations echo previous findings by Epstein and Olsen (1996), who had argued that the successful implementation of RBM depends on the organization's ability to establish and maintain a management culture whose sole focus is in results. Still, on culture, senior managers should be visibly seen propelling the RBM regime. According to Golinelli (2010), experimentation and 
innovation need to be supported, and both managers and staff should be given the opportunity to demonstrate their proven RBM practices, as well as the programs they are accomplishing using those practices. For new organizations introducing RBM, senior management staff will more often say the right things to be seen as supportive (Kusek \& Rist, 2004). However, the actual actions could suggest otherwise. In this regard, there is likely to be an inconsistency about RBM, and this can undermine the organization's success.

While reviewing the New Zealand experience, Norman (2002) notes that RBM systems only influence the organizational behaviour when the top managers opt to use the available information throughout the organization. A Canadian delegate making a presentation at the World Bank roundtable in 2006 emphasized on the need for managers to "walk the talk" in delivering on the RBM approaches. This involves senior management staff respecting their managerial freedom as part of an RBM culture, and supporting other subordinate managers who may be experimenting with new RBM approaches.

RBM systems are mainly motivated by two fundamental principles that include performance reporting or accountability and performance learning or improvement regarding efficiency and effectiveness (Binnendijk, 2000). It is these two pillars that have transformed RBM into a critical tool that assist policymakers and decision-makers in tracking organizational success (Meier, 2003). As a basic principle, organizational learning serves as the major motivation behind the successful adoption of the RBM approach in the most effective organizations (Eriksson \& Kovalainen, 2015). RBM facilitates organizational learning by channelling performance information to policymakers through feedback loops from continuous performance evaluation and audit activities (Farrell, 2009). This process creates ideal opportunities for learning at the individual, group, and systemwide levels, thus continuously transforming the organization in a direction that increasingly satisfies its shareholders (United Nations Educational, Scientific and Cultural Organization, 2010).

When it comes to performance reporting, simplicity has been identified as a fundamental tool for successful RBM implementation (Kvint, 2015) Instead of focusing on the core set of expected results, most successful organizations have a tendency to design complex results chains with finely differentiated outcomes, outputs, and impacts. This approach increases the number of performance measures and indicators needed to produce reliable performance data by an exponential factor. However, a study by United Nations Development Programme (2007) revealed that the best ap- proach is to keep the indicators and results for the vital few who can continuously monitor the entire results chain and transform the internal audits and evaluation into possible learning opportunities.

\section{Theory of change in RBM}

The theory of change ( $\mathrm{ToC}$ ) is one of the most thorough approaches applicable in the implementation of RBM. Holistically, RBM is composed of three components that border on performance in the broader sense, performance measurements, and strategic planning. In the strategic planning, the organization identifies clear and measurable objectives, which are connected to precise indicators. The objective is to target specific milestones. The fourth aspect of holistic RBM is to develop a performance monitoring system that is in turn connected to the reviewing, analyzing, and reporting of the actual results (outputs, outcomes \& impacts) which correspond to the set targets. The findings are then evaluated to facilitate organizational learning.

Management uses performance information collected to improve accountability, resource allocation, learning, and other decision-making processes. In strategic system approach in RBM, ToC can be perceived as the strategy for change that seeks to explore the major items/resources that need to be installed for developmental change to occur (Tomar, 2009). It involves the contribution of partners in the program management such as the partnership between donors and aid recipient countries. It outlines the role of the partners and non-partners in executing change in an organization. ToC approach in RBM draws the intervention methods necessary to cause change (Nigel, 2011).

One of the models that seek to explain ToC is the value, support, and capacity model. In the value component, it seeks the specific benefit of solving a problem, i.e., the benefit to a country or region for executing relevant changes through intervention. The second component is the support required for carrying out the particular intervention. The support, for instance, can be in the form of government or Non-Governmental Organization (NGO). Support can also be from within the organization such as the support from the board of directors. The third component of the model is the capacity and comparative advantage. This component queries the capacity and comparative advantage gained by carrying out the interventions (Tomar, 2009).

\section{Limitation in the Implementation of RBM}

The challenges derived in the implementation of RBM stem from the tensions and trade-offs between accountability and management of results as required in the management 
paradigm (Gianni, D'Ambrogio, \& Tolk, 2014). RBM is the prevalent management model in the public sector and NGOs (Moynihan, 2006). In these organizations, performance information is not part of the budgeting, constraining the effectiveness of RBM in its implementation. In the initial stages of the application of RBM, the results are based on the actual goods and services produced, rather than the outcomes (Binnendijk, 2000). Currently, there has been an increased focus on the outcome of the RBM results; the challenges are more complex than in the initial stages of implementation where the outputs were the major focus (Binnendijk, 2000). The performance assessment at the output information may be less challenging compared to assessment of outcome information (Moynihan, 2006).

It requires fundamental changes in the organization to integrate performance information in the management and budgeting (Moynihan, 2006). This requires evidence-based outcome focus that will, in most cases, lead to significant and fundamental changes in the way organizations are managed (Moynihan, 2006). The implementation of RBM will need organizational behavioural changes in the delivery of programs and services. As noted by Behn (2001), reorientation of the organization to adapt to RBM will change the operational management and personnel assessment down even to the strategic planning and budgeting in the organization. This will be a cultural change where performance information becomes essential in the management of the organization (Behn, 2001).

The successful implementation of RBM may take relatively slow progress fuelled by consistent effort. The long period of implementation can be disrupted by changing priorities - as key people along the path of implementation move on, and there is an alteration of governance structure in the organization. This will lead to relearning that will further extend the time required for full implementation of RBM (Behn, 2001). Apart from the temporal resources invested in the implementation of RBM, there are also additional costs that hinder its RBM implementation, especially in small organizations with comparative low returns (Behn, 2001).

\section{Best Practices in RBM Implementation}

To successfully implement results-based management in an organization, it is imperative to identify RBM's best practices. Ideally, the best practices are based on six principles. These include promotion and support of a results culture in the organization, fostering senior-level leadership in the RBM, building results frameworks within the ownership at all levels, assessing and developing a sensible user-friendly
RBM information system (Mayne, 2007).

Other principles include building an adaptive RBM framework, where there are regular assessments and updates as well as using the results information in the learning and management of the organization to instill a reporting and accountability culture in the organization. In fostering senior-level leadership in the organization, the senior managers are supposed to consistently lead and support RBM through their policies and actions (Mayne, 2007). This should include but is not limited to, supporting RBM in resource allocations, fostering RBM champions and managing the expectation of RBM (Mayne, 2007).

Promotion of an RBM culture may include the provision of formal and informal incentives, which seek to support RBM practices (Mayne, 2007). This calls for more autonomy for the managers to manage the results and accountability programs under their leadership. The accountability framework should put into consideration the challenges of managing the outcomes and impacts of RBM (Mayne, 2007). Best practices involve instilling a result culture that encourages learning from past performances and adjusting accordingly after identifying various modalities such as regular forums and results in information sharing (Mayne, 2007). This can help in the identification of areas of improvements through an in-house RBM capacity. In-house professional RBM capacity can foster continued training of staff and even managers on the best practices in the adoption of RBM (Mayne, 2007).

A culture of results is also supported by in-house professional RBM capacity and through ongoing training of managers and staff in RBM thinking and practices. Ultimately, the underlying culture of results is a clear and shared vision of the value of results information and the role it should play in managing the organization, and of the roles and responsibilities of the various stakeholders in RBM. In establishing a results orientation, any firm will need to develop and agree on a strategic results framework, outlining the organizational key objectives and the strategic system approach in meeting the objectives.

The second layer of the RBM framework structure is developing results frameworks for the programs. This involves outlining the expected specific activities and how they will lead to the achievement of the intended results for each program (Mayne, 2007). There is considerable guidance available on developing such results frameworks and the sequence of results underlying them. At both the organization and program levels, it is good practice to address the risk faced in meeting objectives. Results, which are focused on planning, imply that realistic and clear objectives for pro- 
grams are identified and that there are performance expectations set out for each program (Mayne, 2007).

Best practices emphasize the need to get insights into the types of expectations/targets being set - predictive or stretch targets. In addition, it is important to consider a multi-year strategy for establishing expectations as experience in assessing RBM is gained (Mayne, 2007). Expectations for RBM are thus based on established baselines, past trends, and available resources; and involving all those engaged in the managing and delivery of programs (Flint, 2002). Setting indicators to track performance completes the RBM framework structure. It is important to set a manageable number of indicators, which can easily be measured, without missing critical performance indicators. This is to ensure there are no unintended distortions in program delivery.

Lastly, it is important to build ownership for the various results frameworks established in an organization (Flint, 2002). Without ownership, there will likely be little use made of the information gathered from the results. Best practices include building a buy-in, through the involvement of those using the framework and linking the frameworks with work plans. In addition, ownership of RBM framework can be done through the building of a solid base for RBM using champions and pilots; and ensuring that the $\mathrm{RBM}$ regime is relevant and useful to managers. It should also be flexible enough to accommodate various types of programs (Flint, 2002).

Setting up well thought-out results frameworks is a good base, but without them actually measuring and analyzing the results being achieved, the RBM regime will not deliver much (Flint, 2002). There is an extensive experience available in measuring results and best practices. It is necessary to make use of this experience. Measurement and analysis of "fit for purpose" and the appropriate use of evaluations to complement ongoing performance measurement, are viewed as best practices (Mayne, 2007). In addition, steps should be taken to control the quality of the data being gathered. Both results and the costs of achieving the results need to be measured. There is also the need to assess, as best as possible, the extent to which a given program has contributed to the results being observed (Mayne, 2007). The results data and information gathered as part of the RBM regime will be part of the information system of the organization. The need to customize the results information within the IT system and to make the RBM system userfriendly is underlined as a best practice (Mayne, 2007). Using the results information to help manage the organization and its programs are the aims of RBM implementation.
There is a tendency for results information to be mainly focused on reporting. Thus, best practices are geared towards perceiving results as information rather than for determining the decision-making process. This leads to the balancing of the managerial requirements and the needs of the corporation in the RBM practice (Flint, 2002).

Most organizations apply results information for reporting on how well they are doing, especially to external audiences such as regulatory bodies. This is usually aimed at having a credible performance story about the accomplishments and to even point out underachievements. Finally, results information can play a useful role in the accountability processes in an organization, informing on the results achieved through the use mechanisms such as results-based performance agreements and balanced scorecards (Mayne, 2007). The last principle speaks to the need to regularly review and update the RBM system (Mayne, 2007). Best practices include annual reviews and the willingness to change the RBM system, keeping track of problems within the RBM system by getting feedback from users of the system, and after few years, undertaking an evaluation of the RBM regime (Flint, 2002). Firms working to enhance their RBM capacity should be able to find a few specific suggestions for improvement in the report. For a particular organization, the prominent best practices depend on the robustness of its RBM and its limitations (Flint, 2002).

The process of monitoring and evaluating the RBM requires the evaluator to take a written assessment of the gaps and successes in the program management. This results in the involvement of the evaluator in the learning, decisionmaking, and accountability process of the RBM. The reliance on the evaluator makes the process of results-based management evaluation non-standardized since each evaluator has a unique perspective on the program management. It is difficult to have two evaluators with the similar views on the RBM. Therefore, the actions taken after the assessment of the RMB model will differ depending on the individuals assessing the program management (Flint, 2002).

There is a dearth of understanding of RBM as a resultsfocused approach in its implementation in the organization. As a consequence, and in some cases, institutions are unable to identify and differentiate outputs from out-comes strategically. The reporting of outputs and outcomes, therefore remain an area of weakness in the implementation of RBM since the field managers, project managers or the logistical personnel lack the capacity to identify outputs from outcomes (Golinelli, 2010). The project manager may, at times, not emphasize the significance of results since they perceive outputs to be out of the scope of the results-focused strategy 
of management, thereby ignoring the assessment of results early in the program management. Most focus is placed on the final outcomes, which are tangible to the program managers.

Effective RBM implementation may not occur without the inclusion of partners and stakeholders who understand the objectives of the organization. Effective RBM application is fostered by an explicit commitment of existing partners (United Nations Educational, Scientific and Cultural Organization, 2010). The partners in RBM should be involved in the planning and reporting of the RBM process. Effective RBM practice, especially in the not-for-profit organization, will not occur in the absence of the developing partner. The presence of a developing partner increases the commitment to the plan in the program management. RBM monitoring and reporting involving only one side of the partnership may not bear substantial and relevant results, since the outputs and outcomes are valued differently by the partners (Cross, 2011).

Another challenge in the RBM practice is the identification of realistic and unexpected results. The identified and targeted results should be realistic so that there is a successful results-based strategy in the program management (Itell, 1998). Results projections should be based on achievability rather than the ambitiousness of the results, which in the end, will be unsustainable from a management perspective. The identification of unexpected results is achieved through precise definition of expected outcomes, outputs, and impacts (Kvint, 2015).

When a donor requires targeted results to be part of a proposal, it is important for those results to be evaluated on how achievable they are, not simply their ambitiousness. Executing agencies and their implementing partners on the ground also regularly ignore the identification of unexpected results. The need to define expected outputs and outcomes and impacts at the planning stage can catalyze the focus of those implementing a project into achieving those specific results. Unexpected results, which may be very significant, end up being ignored or downplayed if they do not neatly fit into the original results framework (Kvint, 2015).

\section{Meaningful Stakeholder Participation}

RBM cannot be effectively managed without key stakeholders - beneficiaries, development partners, and donor agencies - involvement. Stakeholder participation must be relevant so that realistic assessments can be achieved without significant challenges. Some proponents push for equal partnerships or equal participation. This is impossible to measure and even the word "meaningful" is open to discourse Grappling with the challenges of defining, implementing, and ascertaining meaningful stakeholder participation is vital to RBM, but it is also one of the hardest things to operationalize effectively.

\section{Appropriate and Effective Indicators}

Appropriate and effective indicators are critical for measuring success and feeding project learning. Constraints on time and resources often lead to the selection of simplified, easily gathered quantitative indicators that do not measure results as deeply as they could, especially at the outcome level. For example, the mainstreaming of gender equality has made the inclusion of gender issues and accompanying indicators mandatory in many projects. It is common for projects to select indicators that measure nothing more than the percentage of project staff and participants who are women. While this is important, does it measure the change in gender relations or power imbalances in any meaningful sense? Simplified indicators make it much more difficult to measure meaningful relationships between inputs and results.

\section{Managing Risks}

All project partners need to be able to take informed and timely action to manage risks. Projects must be nimble and flexible enough to adapt to changing conditions over their duration. Are the executing agency, the partner or partners, and the donor agency entrepreneurial enough to make changes in project design and capacity as needs and the environment change over a multi-year period? If not, RBM risks being thwarted, leading to results that are inappropriate, irrelevant or both. This should not be confused with manipulating project ends to meet the capacity or interests of the executing agency or beneficiary.

\section{Limited Focus on Evaluation}

In many cases, there is a limited focus on external evaluation within project and program activity, despite the critical role of evaluation in measuring results and generating learning. Some projects and programs are evaluated annually, but many others are not. An end-of-project evaluation has no influence on project or program delivery. An external evaluation at the $60 \%$ point of a project is also hard-pressed to make a mark. Furthermore, there is often a challenge in maintaining consistency in evaluation. For example, a five-year project or program could have three different donor-agency project officers and two different monitors over that period. Maintaining evaluation consistency in such a context is a considerable challenge. Performance incentives and consequences include: Does an exe- 
cuting agency that reports poorly, with little apparent commitment to or knowledge of RBM, compromise significant potential results? Often the results of not being committed to RBM are not disastrous enough to modify behaviour in advance, and maybe not even so problematic as to affect behaviour significantly after the fact. Similarly, are projects rewarded appropriately for results that have been defined, achieved, and reported through the participation of all stakeholders? Without real incentives for achieving results or consequences for poor reporting and management, the potential of a results-focused strategy is greatly diminished.

\section{Opportunities for Learning}

Results-based management training is focused on employee enhancement processes during which every employee undergoes training by their respective supervisor or the management team of the human resources department of the organization. Pompa (2012) indicated that training is one of the most effective responses to enhance the skill and knowledge of employees and stakeholders responsible for infusing new roles and responsibilities for any tasks being rendered. The purpose of training, as established by resultsbased management, is to promote the productivity of the company as well as to reach its targeted goals on a regular basis. This approach seeks to increase the level of competitiveness of the vulnerable target as they are going to be trained by their respective managers on a regular basis. Training can be incorporated with seminar workshops so that participants will be given the chance to demonstrate all the learned procedures essential for the productivity of the operating organization. The results-based management training will enhance participant ability to be more analytical, curious, and motivated. The goal of training is to improve the level of performance and knowledge of participants. After the training, the impact enables participants to become more productive, reliable, and functional. Indeed, a good reflection of the model.

One of the key roles of RBM reporting is to provide information that can be acted upon. Managing performance requires ongoing learning. The connection between data collection and reporting on the one hand and the incorporation of learning arising from these data on the other is often not made. The demands of day-to-day operations frequently rob organizations of time to reflect on information gathered through monitoring and evaluation, to draw lessons from these reflections, and to incorporate this learning into project management. The result is lost opportunities. The best projects incorporate learning throughout their lifes- pan, leading to effective, efficient, and relevant results for beneficiaries. Many projects simply cannot find the time to do this.

\section{Conceptual Framework in the RBM Approach}

This management strategy facilitates the achievement of strategic goals. In particular, program managers can utilize this model to map out the goals, set deliverables and boundaries, and produce the intended outcome. While the unwanted outcome is at times part of the RBM process, it is within the results-based management plan to accommodate crisis intervention and risk management to tackle or prevent any unintended outcomes in the process. To facilitate the achievement, the RBM method employs several steps that lead towards the intended goal. The method includes assessing the situation at hand, defining the possible causes for the current situation, envisioning a new scenario, planning it, and initiating the project with a change management plan in hand. The retrospective stage includes lessons learned and future goals. While in several circumstances unintended results cannot be avoided, they, for the most part, serve as compelling and frequently invaluable contributions to lessons learned. Results-based management is a strategy that primarily anchors its logic on feedback to achieve the goals at hand. Those who contribute to the results, be it management or other organizational actors, are doing so indirectly or directly and, as a result, there is a process created that facilitates an outcome. The outcome may be in the form of a new product/service, a change, or an impact/contribution towards the goal at hand. The information gathered from these strategies is then used to account for future needs within the program. RBM is a strategybased control mechanism similar to third-generation balanced scorecards (Lawrie, Kalff, \& Andersen, 2005).

RBM is often used by organizations such as the UN and the International Committee of the Red Cross (United Nations Development Programme, 2007). It is used for commercialbased organizations as well, including the Asian Development Bank. It will also be useful to include utilization of RMB by other establishments. For instance, the U.S. government uses Management By Objective (MBO) - similar to RBM - in its government departments. The results approach is also used in program development, and it has been implemented since 2000 (Global Affairs Canada, 2008). All program cycles began with the use of RBM, and it has not proven to be an efficient way to continue the development and acceleration of program management and noncommercial management programs. If that approach failed as indicated here, what alternatives did they use? And, how 
did that solve their problem?

To apply RBM, the program manager must assess the current situation and decipher the best possible attainment for the betterment of the program or project. The next step is to think about its causal and relational effects, followed by the vision of what is required to achieve the purpose. The planning stage includes the assignment of roles and responsibilities, as well as resources. The execution stage involves the need to adapt to external and internal circumstances to attain the goal. The last stage in the RBM framework involves the retrospective lessons learned, accumulation of organizational assets, and the enterprise environmental factors that will allow for future similar endeavours to be a success.

\section{Definitions}

Results-based management: RBM is the form of management that is tailored for results (Sevaldson, 2011).

Results oriented: The design and management of a project in a way that will assure the continuous focus on outcomes and their achievements (Sevaldson, 2011).

Results-based monitoring and evaluation: This encompasses the constant focus on collecting and analyzing the data available to predict outcomes better and measure the progress of the expected issue.

Continuous adjustment: This involves the collection of data and its subsequent assessment throughout the life cycle of the project and the adjustment of its operational standards, implementing the strategies to maximize the probability of the result (Sevaldson, 2011).

Managing risk: Risk management in RBM entails the identification of risks about the expected outcomes and attaining the necessary resources to overcome them (Mayne, 2007). Participatory approach: This is the constant and active participation of stakeholders, which includes beneficiaries as well as intermediaries, donors, and implementers.

Crosscutting theme integration: During the results-based management cycle of planning, design, and implementation, issues such as equality, sustainabi-lity, and governance are considered crucial parts of the process (Sevaldson, 2011).

\section{RESEARCH METHODOLOGY}

The methodology seeks to explain how the research will be performed and the data collection process implemented for a given study. Theoretical consideration and the theorybuilding process that will be undertaken in a potential study is provided.

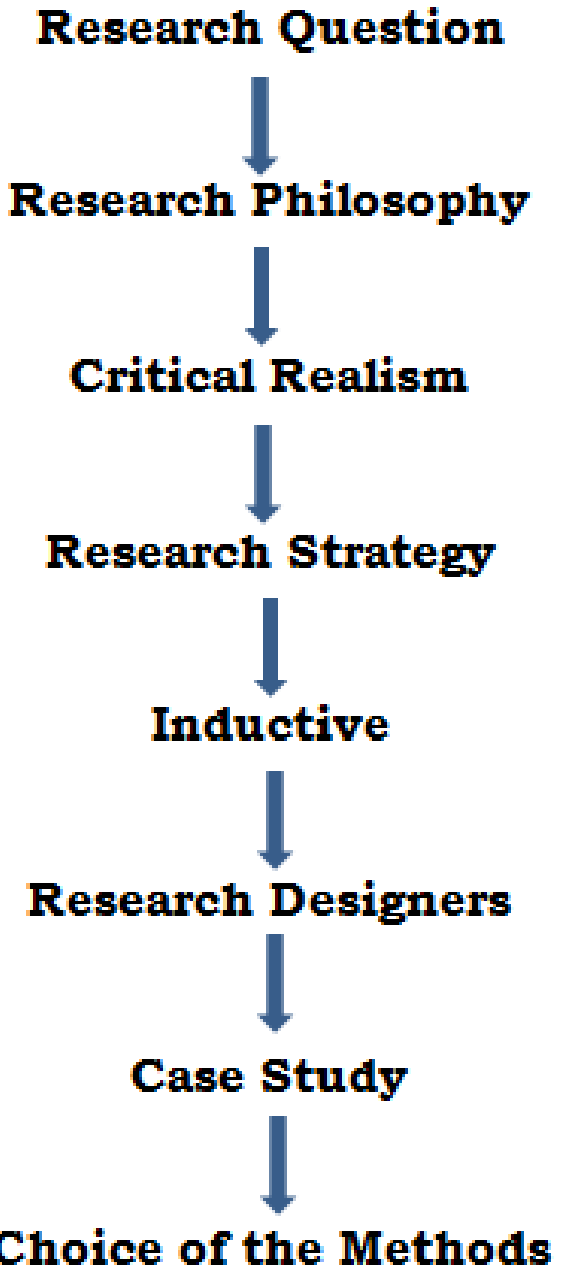

FIGURE 1. Results-based management methodology

The philosophical assumptions underlying this study are based on critical realism. This study seeks to establish the mechanisms applied in results-based management and the structures that are required via a life case study of the UN organizations, presented later. In critical realism ontology, three aspects are considered, the real, the actual, and the empirical. To understand the application of RBM in the case study, this study combines all the three aspects of an insightful analysis.

The study also applies abstraction and concretization, including an induction approach where the analysis progresses from the actual level to the empirical implementation of RBM in the case study.

\section{Theoretical Consideration}

Theoretical consideration seeks to reinforce the choice of theory in any research. For RBM, monitoring and evaluation is applied to explore the case study. Systems and a variety of theoretical perspectives could be applied. 


\section{Theory Building}

This study will seek to build theory through triangulation by engaging multiple paradigms in understanding the implementation process of results-based management in an organization (Nigel, 2011). Designing a "state of the art" RBM system is not only difficult to achieve but also complicated by the lack of a universally accepted "gold standard". Reasons for these complications vary. Some include the inadequate effectiveness, limited quality assurance, and overall oversight mechanisms. There are also other exacerbating factors like the inability of program designers to effectively manage the unreliable and incomplete availability of required data sets. Another challenge faced by designers is the ability to produce a program document within an unrealistic timeline. An attempt to overcome this deadline crunch exposes the team to myriads of vulnerabilities.

Team concordance, harmony, and complementarity are also other compelling issues that must be addressed by the team. In some cases, the program designers spend a significant amount of time trying to establish consensus; sometimes on trivial issues. For example, during one assignment, I remarked several instances where the team could not agree on where to incorporate some performance indicators in a strategic framework. And this is just the tip of the iceberg.
In light of some of these shortfalls, I am proposing in Figure 1 a "road map" that I believe will serve in mitigating some of the system deficiencies that continue to erode attempts to produce a useful and effective RBM system.

\section{Research Design}

This life case study explores a case study of how resultsbased management has been used by UN organizations in project and program interventions. The study investigates the different frameworks that have been utilized in the successful implementation of RBM. The case study allows for the use of multiple data collection methods to explore the case (Creswell \& Creswell, 2017). The choice of these methods relates to the nature of the case and the research objective. In exploring the life case study, this work seeks to answer the "how" and "why" in RBM implementation through an explanatory type of research.

In explanatory research, the aim is to uncover the causaleffect-linkage, and in this context, the effective contribution of RBM implementation in enhancing the effectiveness of UN organizations. Descriptive research has been used to identify and collect information regarding the implementation of RBM. Selected UN organizations have been used due to the rigorous implementation of RBM in a variety of projects within the organization over the years.

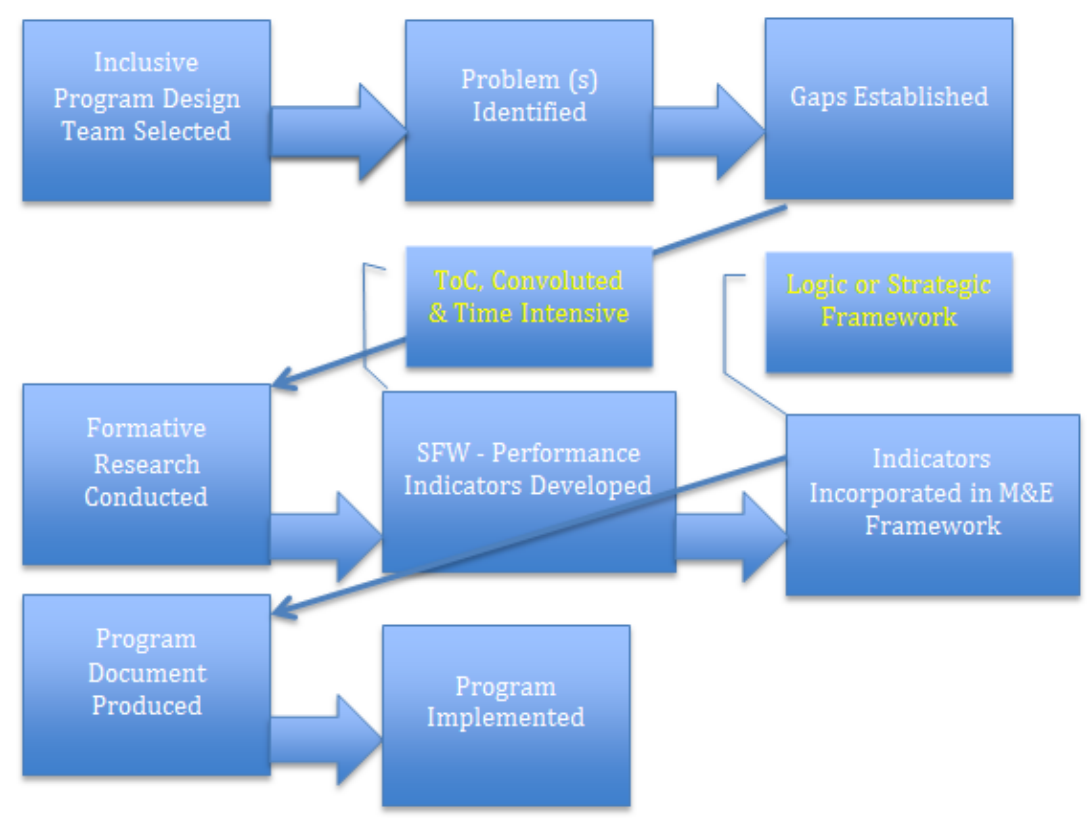

FIGURE 2. Proposed RBM system methodology algorithm

In a related World Bank article, Roberts and Khattri (2012) highlight some of the salient and crucial elements to be considered and included in an effective and useful RBM approach. The authors also emphasize the role and impor- tance of ToC and how the strategy helps in improving program results. And using the concept of ToC, the authors further elaborate on how it helps in establishing the necessary and relevant pathways required in effectively and genuinely 
mapping the hierarchical causal links amongst the different result levels of a strategic framework. They also included a modified sample case study (Figure 2 as an illustration of a "success" story.

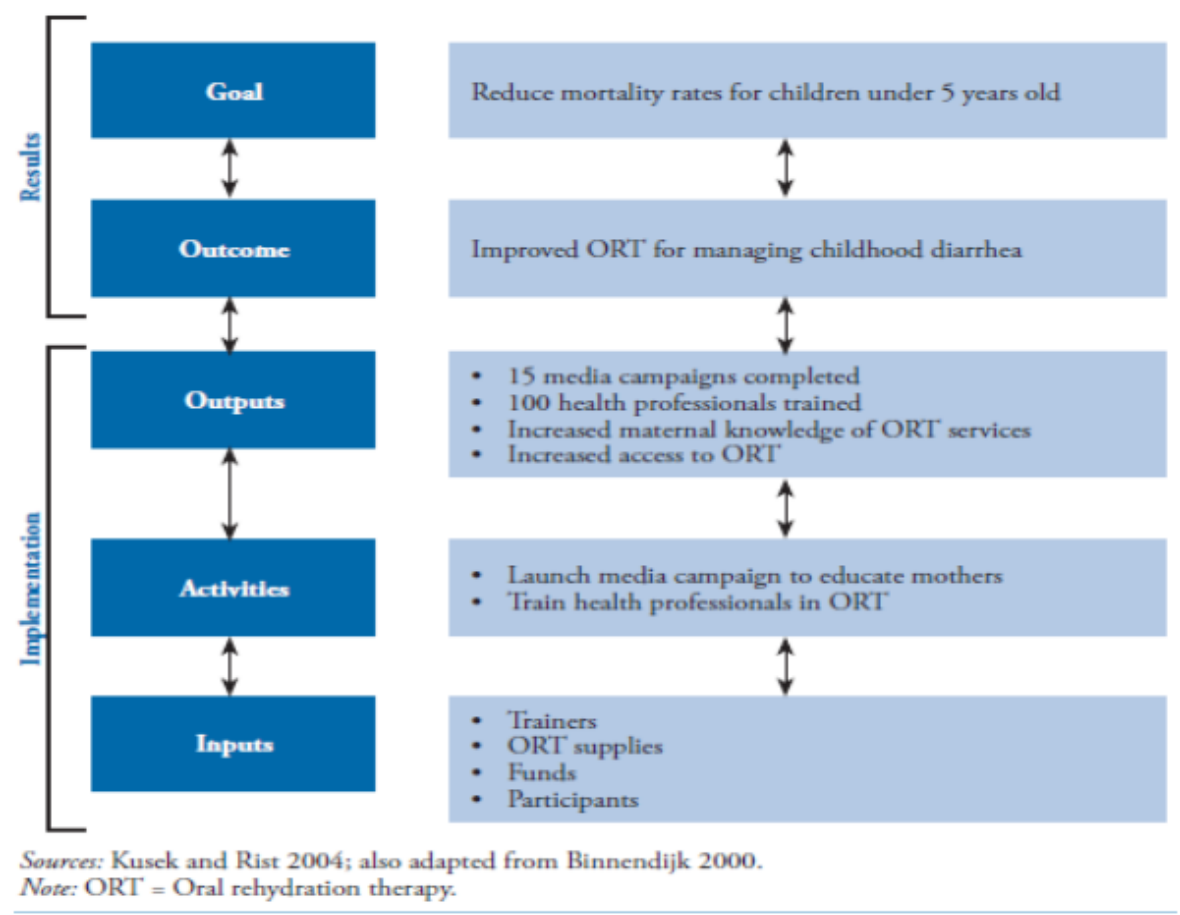

FIGURE 3. Sample program logic to reduce childhood morbidity through the use of oral rehydration therapy

\section{Scope and Significance of RBM Frameworks}

The vital role of a RBM system could never be adequately emphasized. RBM systems are universally acceptable strategies. They give program managers and different stakeholders a better sense of direction, an evidencebased management approach, a consensus-driven decisionmaking process, an opportunity to make informed and effective decisions, and ultimately, an invaluable, necessary, and sufficient pathway to success.

\section{A Better Sense of Direction}

The concept of RBM is not new. Indeed, it underlies all the efforts humans consciously undertake to achieve desired results. For example, countries go to war to win, parents bring up their children to be law-abiding citizens, children go to school (elementary, middle school, high school, community college, university) to successfully graduate, farmers plant crops in anticipation of good harvests, people go to gyms to lose excessive weight, businesses are established in order to make profits, and so on. All these interventions have in common the objective of transforming inputs into intended results (outputs, outcomes, and goals) or SO.

The universality of this concept makes it a useful basis for efforts to streamline and optimize proposed interventions. From a program development perspective, definitions have limited RBM to the key results presented in a relevant PDF. This approach (while correct) seems to limit the importance and significant scope of RBM. In this document, the definition of RBM has intentionally been expanded. It has also been deliberately presented from a generic perspective. Such a strategy creates room for every stakeholder and facilitates portability - lessons learned. It also enhances the versatility of this concept.

Thus, I have attempted to define a more holistic meaning of RBM. RBM can be considered as a hierarchical framework of mutually complementary components (PDF, M\&E, data management) and MIS with synergistic dynamics that collectively yield intended objectives. The beauty of RBM is that there is no absolute "goal standard". The unavailability of an acceptable "turn-key" system is a confirmation of the dicey nature of RBM. Each intervention is different, and as such RBM serves as a unique framework with a common potential of promoting efficiency and accountability (effectiveness). Effective and evidence-based performance monitoring contribute substantially to the achievement of RBM. It is my hope that such a document will serve a wide audience on the one hand and facilitate program design and implementation on the other.

While the target audience remains quite broad - bilateral 
and multilateral agencies, academic institutions, Program Implementing Partners (PIM), and academic institutions it is also my hope that this document can be used by anyone interested in an improved understanding of the concept of RBM. To this end, I have tried to minimize utilization of technical jargon to the extent possible. Such an attempt will hopefully expand the target audience base and generate more interest, especially amongst the members of the program development communities. Finally, the current requirement for accountability and results by donors has provided compelling evidence of the need for information of this kind.

\section{Life case study - RBM implementation in the United Na- tions organizations}

The United Nations applies the results-based paradigm in a different perspective, where different terms and terminologies are used to represent the results-based approach in the management of institutions related to the UN (Bester, 2012). Also and more specifically, in the UN system, there remain significant differences in the thematic application and implementation of RBM amongst the various agencies. For instance, in the United Nations Children's Fund (UNICEF), planning and management replaces a resultsbased approach while the UNDP uses results-based budgeting. In the Food and Agricultural Organization (FAO), the results-based approach is substituted by the strategic framework, or even enhanced monitoring and evaluation regime.

The existence of different conceptual frameworks in the UN describing the results-based approach complicates the communication across the organizations. It points to the different views, of RBM in the organization structure usually aligned with the agency's mandate and objectives. While some organizations perceive RBM as management practices, others perceive it as budgetary practice and others may objectify it as a bureaucratic requirement of the organization. In all, the organizations operating under the UN funds and programs, such as UNFPA, WFP, and UNDP, have been found to have a systematic and methodical implementation of RBM (Bester, 2012).

These organizations have approached RBM as a management practice and have also learned from the experiences of other organizations. This has made it possible for them to build an effective and solid system of management. It is important to note the significance of conceptual frameworks in the proper implementation of RBM. This has been a major challenge in the perspective of RBM in the different UN organizations and the eventual application of the manage- ment practice (United Nations Educational, Scientific and Cultural Organization, 2010). Conceptual frameworks lay the ground for the tools and terminology for harmonizing RBM within the organization. The conceptual framework seeks to link RBM to the business and operation of an organization.

A conceptual framework is a time-bound coherent strategy for implementing RBM. In the context of the UN, there is the lack of a coherent and holistic approach to RBM as a pure management practice (Bester, 2012). The advancing of RBM has mainly concentrated on the format and vision without much regard to the effective translation of RBM to improved work process to support the administrative, financial, and information system within the organization (Bester, 2012). Many of the bottlenecks in the application of RBM in the UN have been found to reside in the understanding of conceptual issues amongst the managers (Mayne, 2007). Where there is a clear conceptual framework of RBM, the organization is committed to the implementation and institutionalization of the RBM process. In conclusion, the presence of a clear conceptual framework illustrates a broad management strategy of the organizations.

Another important facet of RBM in the implementation process at the UN is the respective responsibilities of the partners in the RBM. Effective implementation of RBM calls for a shift in the operational modalities of an organization. This presents a significant challenge for the member states of the UN to adapt to an outcome-driven programming and budget from the input-driven process of program management in an effective an efficient manner (Bester, 2012).

Being a new form of management practice, RBM adoption has been slow in most of the organizations under the UN as stakeholders simply adapt to the system management to avoid setbacks and work efficiently (Bester, 2012). The culture of RBM can only be consistently harnessed when the ownership of the programs is fostered and the accountability in the organization is promoted (Bester, 2012). The RBM at the UN promotes a culture of trust and understanding between the member countries (ownership,) and the UN Secretariat (managers) under an environment of trust in micro level management in the RBM settings.

The UN also adopts a key principle in RBM practice, which is vested in the long-term objectives of an organization. The UN system has acknowledged and recognized the need for strategic frameworks that provide the vision and overall direction of the organization. This is based on both shortterm and long-term results in RBM. The sum of the longterm organizational objectives contributes to the overall or- 
ganizational goals such as the UN Millennium Declaration (Mayne, 2007). This helps the organizations to identify its priorities in the implementation of its programs.

The goals and sub-goals, which contribute to the priority goals, are defined, and in the RBM, they constitute the critical results to be achieved or assessed in the organization. Therefore, the objectives of the UN organizations are precise, clear, and verifiable, so that the performance assessment process is effective in the long-term (Bester, 2012). Essentially, the desirable traits of the statement of objectives in RBM entail a statement of results, which is devoid of actions or means, with precise, simple, measurable and logically consistent objectives, across all levels of the organizations that reflect the causal-effect-linkage.

Alignment of the programs with the organizational goal is a principal component of program management at the UN organizations. This helps in combining results so that they can constitute a major medium-term outcome of the organization. The alignment of programs with the organization objectives is through a cascading process that seeks to set the sub-objectives at the operational levels of the organization. For example, the World Health Organization (WHO) programs are grouped into three levels, global, regional, and country, and the specific objectives are lumped together to form the overall objectives of the organization (Bester, 2012).

The UN implementation of RBM demonstrates the flexibility of the RBM approach to management. Resources are aligned to the long-term objectives of the UN organizations. This gives birth to the results-based budgeting as a variation of RBM, which harnesses organizations resources to meet the desired objectives by demonstrating the link between results and resources in an effective RBM approach (Bester, 2012). The RBM framework allows for the shifting of resources from underperforming and obsolete programs, to more efficient and relevant programs considered high priority. In RBM in the UN, effective cost accounting is linked to the resources, which are then tied to the organizational goals. Performance assessment is thus, performed in tandem with the budget cycle of the UN organizations. The predictability of available resources in the UN system acts as an important tool in aligning strategic planning with the results. It helps in integrating the multi-year funding frameworks to the programs objectives, resources, budgets, and expected outcomes (Bester, 2012).

\section{RBM and the Accountability Framework}

The Results-Based Management and Accountability Framework (RMAF) is intended to serve as a blueprint for man- agers to help them focus on measuring and reporting on outcomes throughout the life cycle of policy, program or initiative. Managing for results is not completely new to businesses organizations but what makes the current approach different is the determination to make RBM the driving force behind the organization's institutional culture and practice - and to develop and apply a corporate methodology for this purpose. When it comes to results-based management, balance is key to prevent the process of defining and monitoring indicators from becoming a major workload.

In this regard, balance requires that the definition and use of indicators have to be taken seriously for credible and effective assessment, learning, and accountability. On the other hand, care must be taken not to overinvest in results measurement and indicators. If the measurement is emphasized too much there is a risk that managers will be motivated to undertake certain activities simply because measurable results can be achieved. In the process, they may be diverted from less measurable, but ultimately more fruitful, development interventions.

It is noteworthy that the outcomes and outputs furnished within the strategic results framework by operating units should reflect the key results against which managers wish to be assessed. However, because outcomes are not the result of one single actor's intervention, enforcing individual and personal accountability with respect to the substantive attainment of outcomes would be unreasonable. In this regard, the measurement of results is not an isolated activity. Rather, the process of measuring results begins with the design of policy, program or initiative, and its evolutions. Different results-measurement activities occur at different points in time, but always as part of the ongoing management of policy, program or initiative. This continuum runs from the initial consideration of performance measurement, through performance monitoring, to formative and summative evaluation. While managers can be held accountable for ascertaining that outcomes are monitored, their full accountability can be applied only to outputs. In a situation of shared accountability, it is important that responsibilities and performance expectations be defined. Managers, while not being held accountable for the achievement of outcomes, are expected to report on progress against intended outcomes.

\section{RBM and Development vs. Management Results}

As a critical step in the evolution of logical framework approaches, RBM attempts to respond to some issues of the Project Cycle Management (PCM) and Logic Framework Ap- 
proach (LFA) methods. People often ask what the difference is between PCM or LFA and RBM. In a sense, it can be said that RBM is PCM done right. It provides more tools and directives on what should be done to ensure that project design is performed in an inclusive and participatory way, and to make sure that one takes into consideration any assumptions and risks. The latter being defined as internal and external factors that are likely to influence project implementation and outcomes.

RBM is also critical of many donors' focus on inputs (funds and resources) and activities, and promotes a shift towards the results of the project: its tangible outputs, its effects, and its impact - that is the 'results' part of RBM. As for the "management" aspect, RBM provides some tools to monitor the performance of the project. In many projects, there continues to be a tendency to emphasize work plan achievements with little regard for how these achievements contribute towards intended results. Some of the salient questions that need to be answered include: Are you getting the results you wanted? How can you be sure? How many resources do you use? RBM can provide an answer to these questions.

Compared to its predecessors, RBM also makes sure that the context or environment in which one is working is dynamic, relevant, and influences the project - in positive ways but also in negative ways. RBM stimulates the project manager and other relevant stakeholders to think about assumptions and risks, not just at the project design stage, but also over the whole course of the project life cycle.

\section{DISCUSSION}

This work has discussed the approaches for effective strategic systems implementation including the CARROT-BUS strategy, ToC and the results-based management. The ToC contains three components that border on performance in the broader sense of RBM, performance measurements, and strategic planning. In the strategic planning, it identifies clear and measurable objectives, which are connected to precise indicators; a process that is accomplished through the identification and establishment of relevant, reliable, valid, and compelling pathways leading to intended and sometimes unintended results.

The ToC approach in RBM includes developing a performance monitoring system that is linked to the assessment, analyses, and reporting of the actual results, which include outputs, outcomes \& impacts. The ToC stipulates that the findings of the RBM are to be evaluated to facilitate organizational learning. Management uses performance indicators to manage accountability, resource allocation, learning, and other decision-making processes. Ideally, the $\mathrm{ToC}$ is a strategy for change that seeks to explore the main resources that need to be in place before change can occur through effective interventions.

The limitation of RBM as a strategic program management approach has also been highlighted, identifying the challenges and possible opportunities for improvement in the implementation process. The limitations arise from the trade-offs between accountability and management of result in the management paradigm. In most cases, there is a lack of incorporation of performance parameters in the budgeting and management of public and not-for-profit organizations, constraining the successful implementation of RBM. Processes are emphasized more than the output, outcomes, and impacts in applying RBM. This leads to an increased focus on the organization's resources that will lead to short-term outputs, creating imbalances in the three result levels. Thus, performance assessment at the output level may be less challenging compared to assessment of outcome level. Fundamental changes are required in the organization to integrate performance interventions in management and budgeting.

\section{CONCLUSION}

In conclusion, this paper has explored the concept of results-based management as a management approach intended to improve results in a three-thronged model: Short, medium-, and long-term; types of results - outputs, outcomes, and impacts. The concept is particularly prevalent in public and not-for-profit organizations. The analysis has focused on getting an insight on the results-based approach in program management and its application in a selected case study. The UN study explored the application of RBM in UN organizations such as UNESCO and UNDP amongst others, with a focus on the global, regional, and country-based implementation of RBM. The paper critically reviews the advantages of RBM in strategic program management at the UN organization and the significant bottlenecks in the implementation process.

The paper draws important lessons in the implementation of the results-based management model stemming from the introduction of changes in the RBM framework and the incentives for motivating the management to adopt resultsbased management. Research findings note the existence of RBM on policy papers, with no minimal impetus to practically implement it, despite its potential benefits in improving the organizational performance. The life case study focused on the UN and the modalities for the implementation of RBM. The report (Bester, 2016) critically examines 
the successes and the challenges in the implementation process. The section focused on select UN organizations. The role of the managers in drawing objectives in tandem with the logical framework of RBM is highlighted.

\section{IMPLICATIONS}

Research findings recommend the removal of barriers for the implementation of RBM drawn from the UN organizations case study. Such barriers include the incorporation of project management as part of the budget process. The implementation of RBM has considerably been hindered by the parallelism of the budgetary programs and the management process. This implies that organizations are constrained to timely implement the RBM due to budgetary constraints. And finally, for RBM to succeed, significant paradigm shifts by key decision-makers would be a compelling, appropriate, and necessary way forward. More participatory and inclusive strategies would also contribute substantially to promoting and facilitating the implementation of this framework.

\section{REFERENCES}

Aly, W. O. (2015). A framework for results based management to the public sector in Egypt: Challenges and opportunities. Journal of Public Administration and Governance, 5(4), 23-45. doi:https://doi.org/10.5296/jpag.v5i4.8406

Behn, R. D. (2001). Rethinking democratic accountability. New York, NY: Brookings Institution Press.

Bester, A. (2012). Results-based management in the united nations development system. Retrieved from https: //urlzs . com/ NaCwp (accessed on 16 June, 2017)

Binnendijk, A. (2000). Results-based management in the development co-operation agencies: A review of experience: DAC working party on aid evaluation report. Retrieved from https://urlzs .com/6pqP3 (accessed on 15 August, 2016)

Creswell, J. W., \& Creswell, J. D. (2017). Research design: Qualitative, quantitative, and mixed methods approaches. New York, NY: Sage publications.

Cross, N. (2011). Design thinking: Understanding how designers think and work. California, CA: Berg.

Diamond, M. J. (2005). Establishing a performance management framework for government (No. 5-50). New York, NY: International Monetary Fund.

Downey, M. L. (1998). Making GPRA work for your agency. The Public Manager: The New Bureaucrat, 27(3), 18-19.

Due, A. T. (2016). Using open innovation model to enhance knowledge sharing in Vietnam University. Journal of Administrative and Business Studies, 2(5), 241-247. doi:https://doi.org/10.20474/jabs-2.5.4

Epstein, J., \& Olsen, R. T. (1996). Lessons learned by state and local governments. The Public Manager: The New Bureaucrat, 25(3), 41-45.

Eriksson, P., \& Kovalainen, A. (2015). Qualitative methods in business research: A practical guide to social research. New York, NY: Sage.

Eskerod, P., \& Huemann, M. (2013). Sustainable development and project stakeholder management: What standards say. International Journal of Managing Projects in Business, 6(1), 36-50. doi:https://doi.org/10.1108/17538371311291017

European Commission. (1999). Implementation of the A.I.S.E. code in Europe: Complementary report from A.I.S.E. to the PwC 1999/2000 progress report. (accessed on 15 July, 2016)

Farrell, G. M. (2009). Results-based monitoring and evaluation at the commonwealth of learning. Vancouver, Canada: Commonwealth of Learning.

Flint, M. (2002). Easier said than done: A review of results-based management in multilateral development institutions (Technical Report). Department for International Development, London, England.

Gianni, D., D'Ambrogio, A., \& Tolk, A. (2014). Modeling and simulation-based systems engineering handbook. New York, NY: Sage Publications.

Gibson, J., \& Boisvert, B. (1997). Data-driven performance: Accountability, business planning and performance measurement in Canada. In Conference Board of Canada, Ottawa, Canada.

Global Affairs Canada. (2008). Results-based management. Retrieved from https://urlzs.com/wC14u (accessed on 15 July, 2009)

Golinelli, G. M. (2010). Viable Systems Approach (VSA): Governing business dynamics. Michigan, MI: Cedam.

Groot, R., et al. (2000). Workshop on capacity building in land administration for developing countries (Technical report). International Institute for Geo-Information Science and Earth Observation, Enschede, Netherlands.

Hatch, J., \& Cunliffe, L. (2006). Organization theory. Oxford, UK: Oxford University, Press. 
Itell, J. (1998). Where are they now? performance measurement pioneers offer lessons from the long, hard road. The New Public Innovator, 5(6), 11-17.

Kettl, D. F. (1997). The global revolution in public management: Driving themes, missing links. Journal of Policy Analysis and Management, 16(3), 446-462. doi:https://doi.org/10.1002/(sici)1520-6688(199722)16:3<446::aid-pam5>3.3.co;2 $-\mathrm{y}$

Kusek, Z. J., \& Rist, R. (2004). Ten steps to a results-based monitoring and evaluation system: A handbook for development practitioners. New York, NY: The World Bank.

Kvint, V. (2015). Strategy for the global market: Theory and practical applications. London, UK: Routledge.

Lawrie, G., Kalff, D., \& Andersen, H. (2005). Balanced scorecard and results based management convergent performance management systems: Maidenhead, UK: 2GC. Retrieved from http://tiny.cc/9hgw8y (accessed on 1 August, 2017)

Layyinaturrobaniyah, Masyita, D., \& Sekartadjie, G. (2016). Fundamental and technical analyses for stock investment decision making. Journal of Administrative and Business Studies, 2(1), 1-7. doi:https://doi.org/10.20474/jabs-2.1.1

Mayne, J. (2007). Best practices in results-based management: A review of experience. Retrieved from http://tiny.cc/ 07ew8y (accessed on 1 July, 2018)

Meier, W. (2003). Results based management: Towards a common understanding among development cooperation agencies (Discussion Paper). Canadian International Development Agency, Canada.

Montasser, M. H. H., \& El-Nakeeb, I. (2017). Investigating solid waste supply chain: A proposed framework for achieving the environmental sustainability case study Alexandria, Egypt. International Journal of Business and Economic Affairs, 2(3), 165-172. doi:https://doi.org/10.24088/ijbea-2017-23001

Moynihan, D. P. (2006). Managing for results in state government: Evaluating a decade of reform. Public Administration Review, 66(1), 77-89. doi:https://doi.org/10.1111/j.1540-6210.2006.00557.x

Nigel, S. (2011). Results-based management, intrac. Retrieved from https://urlzs.com/p8SWC (accessed on 15 August, 2018)

Norman, R. (2002). Managing through measurement or meaning? Lessons from experience with New Zealand's public sector performance management systems. International Review of Administrative Sciences, 68(4), 619-628. doi:https:// doi.org/10.1177/0020852302684007

Organisation for Economic Co-operation and Development. (1997). In search of results: Performance management practices, paris, france. Retrieved from https: //urlzs . com/q4CfU (accessed on 15 July, 2017)

Panti, E. M., \& Gempes, G. P. (2018). The mediating effect of risk management strategies on the relationship between attitude constructs and sustainability of banana production in Southern Philippines. International Journal of Business and Administrative Studies, 4(2), 68-77. doi:https://doi.org/10.20469/ijbas.4.10004-2

Poate, D. (1997). Measuring \& managing results: Lessons for development cooperation. New York, NY: Office of Evaluation and Strategic Planning, UNDP.

Pollitt, C., \& Bouckaert, G. (2004). Public management reform: A comparative analysis. Oxford, UK: Oxford University Press.

Pompa, C. (2012). Literature review on enterprise mentoring. Retrieved from http://tiny.cc/fcgw8y (accessed on 21 July, 2016)

Roberts, D., \& Khattri, N. (2012). Designing a results framework for achieving results: A how-to guide. Retrieved from http://tiny.cc/d4gw8y (accessed on 15 August, 2017)

Sevaldson, B. (2011). Giga-mapping: Visualisation for complexity and systems thinking in design. Nordes, 5(4), 45-50.

Silvius, G. (2015). Considering sustainability in project management processes. In A. J. G. Silvius \& J. Tharp (Eds.), Handbook of research on sustainable development and economics. Hershey, PA: IGI Global.

Tomar, R. (2009). Commercial operations management: Process and technology to support commercial activities. New Dehli, India: Global India Publications.

United Nations Development Programme. (2007). Evaluation of results based management at UNDP. Retrieved from https://urlzs .com/D27Vf

United Nations Educational, Scientific and Cultural Organization. (2010). Results based programming. Retrieved from https://urlzs.com/JFB43 (accessed on 15 June, 2016)

Vahamaki, J., Schmidt, M., \& Molander, J. (2011). Review: resuls based management in development cooperation: Riksbankens jubileumsfond. (accessed on 1 February, 2012) doi:https://urlzs.com/SQsM4 
Visser, J. H. (2016). Bases of market segmentation success: A marketing decision makers perspective. International Journal of Business and Administrative Studies, 2(3), 75-80. doi:https://doi.org/10.20469/ijbas.2.10004-3

Willy, S. (2017). Analysis of financial ratios to measure the company's performance in the sectors of consumer goods at Pt. Nippon Indosari Corpindo, Tbk and Pt. Mayora Indah, Tbk. International Journal of Business and Economic Affairs, 2(1), 45-51. doi:ttps://doi.org/10.24088/ijbea-2017-21006 